\title{
A Monolithic (Si) Massively Broadband Non-Reverberant Monopole
}

\author{
Antonio Vilches ${ }^{1}$, Kostis Michelakis ${ }^{1}$, Jeff Hand $^{2}$, Chris Toumazou ${ }^{1}$ \\ 1. Institute of Biomedical Engineering, Imperial College London. \\ 2. Radiological Sciences Unit, Hammersmith Hospital, Imperial College Healthcare NHS Trust, London.
}

\begin{abstract}
A novel monolithic implementation of a Wu-King profiled antenna intended for use in critical time domain / UWB / imaging applications is presented. The simulated device was fabricated on a high-resistivity $\mathrm{Si}$ substrate on top of which a layer of GaAs is grown, segmented and patterned to serve as the radiating element. The device's measured return loss indicates a $-10 \mathrm{~dB} \mathrm{BW}>50 \mathrm{GHz}$ and time domain measurements using a 500pS input pulse prove that the device is practically non-reverberant in air.

Index Terms - Antennas, Biomedical Imaging, UWB.
\end{abstract}

\section{INTRODUCTION}

UWB medical imaging is part of a set of critical timedomain applications that use the Fourier transform of a received pulse to derive spectral content.

Such applications include dispersion studies in biophantoms and tissues [1, 2] and remote / non-invasive, body-probing UWB radar techniques such as remote cardiac monitoring [3] and breast mammography. In the latter, as an example, UWB scattering resulting from differences in tissue permittivity is used to identify internal malignant growth [4-6].

All these applications benefit from antennas capable of transmitting temporally short, broad-bandwidth pulses, with the least degree of distortion caused by reflected energy from the ends of the radiating element. Distributive resistive loading, in one form or another, can and has been used in the past to curtail such reverberations.

Wu King et al [7] published the most adequate nonreverberant resistive profile necessary for the cancellation of such internal reflections, when driven by short broadband pulses. Their work offers a solution for the current in an antenna with an impedance per unit length that is a function of the distance from drivenpoint to end-point, which is represented by an outward traveling wave with no reflections.

And there have since been many attempts to implement the profile by employing discrete / surface mount resistive devices, as detailed extensively in [8].

The work presented here, however, is the fabrication of a monolithic monopole device. This relies on the direct segmentation of a top layer of metallization, in which Nichrome, PolySi, MBE (Molecular Beam Epitaxy) GaAs can all be used, to produce the desired resistive profile, with the accuracy of the profile being directly proportional to the number of segments used. This approach cancels the difficult and often expensive approach that is the tapering of material layers to obtain the required resistive profile [9]. Computer simulations, CST [10], indicated that it was feasible to fabricate a massively broadband $(10 \mathrm{GHz}+)$ monolithic planar antenna, employing a $\mathrm{Wu}-\mathrm{King}$ resistive profile, directly on a high resistivity Si substrate.

The synthesis method employed is revealed in Part II; related FDTD simulated data is shown in Part III; device fabrication is discussed in Part IV and measured results are given in Part V.

\section{SYNTHESIS METHOD}

The synthesis method for the monolithic grid technology antenna resistance profile is presented in Fig. 1.

\section{FIG. 1 HERE}

This method defines a segment to be any portion of the layout enclosed by two strip-columns and a strip-column as any vertical strip (at $90^{\circ}$ to antenna length). In essence, the antenna's electrical length is composed of a series of segments, with the resistance being greater for those segments furthest from the feed point (located on the LHS in Fig. 1). The resistance of each segment is computed with given technology related parameters and a layout is produced for each segment, in series, along the antenna length.

With reference to the resistivity of a length of conductive track or strip given by (1), a series of formulae are 
derived to compute the Segment-Length (2), the resistance of a column-strip (3) and the resistance of a segment (4):

$$
\begin{aligned}
& w=\text { StripWidth } \\
& t=\text { StripThickness } \\
& l=\text { SegmentLength } \\
& \rho=\text { resistivity }
\end{aligned}
$$

$$
\begin{gathered}
R=\frac{\rho l}{w t} \\
\text { SegmentLength }=\frac{\text { TotalLength }}{\text { N.of Segments }} \\
R(\text { Col })=\frac{\rho}{\text { TotalWidth } \times t}(2 x \text { ColStripWidth }) \\
R(\text { Segment })=R(\text { Col })+\frac{\rho}{w t}(\text { StripLength })
\end{gathered}
$$

\section{Simulations}

The Transient solver within the CST Microwave Studio Suite [10] of programs employs the Finite Integration Technique which can be considered a generalization of the FDTD (Finite-Difference Time Domain) method [11]. This solver was used to simulate a device with 19 segments; each represented in the simulator by a block of material with an electrical resistance computed using the synthesis method presented. Each of the 19 rectangular segments is laid edge to edge to make a single continuous track on top of a slab representing the $\mathrm{Si}$ substrate, measuring $1.3 \mathrm{~mm} \mathrm{X}$ $330 \mu \mathrm{m}$. Boundary conditions used were an electrical earth below the substrate and 'open-add-space' all round. The resistive profile of the device, as computed in Matlab [12] is shown in Fig. 2.

\section{Fig.2 HERE}

It is a desired trait in critical time domain applications, such as medical breast cancer detection, that the antenna exhibits a much dampened response when energized by a very broadband pulse. One of the main frequency ranges of interest for such applications is the FCC approved Ultra Wide Band $(3.1 \mathrm{GHz}-10.6 \mathrm{GHz})$ and the device was originally designed with this range of frequencies in mind.

Approximate antenna dimensions were chosen for the middle of the required frequency band of operation and preliminary values for a possible range of $\mathrm{MBE}$ grown GaAs layer resistivities, necessary for the solution of the equations in the synthesis method, were obtained from our collaborators on this project in FORTH, Crete [13].

The segment number, segment width, segment length and substrate thickness were all manually optimised, reiteratively, in Matlab and CST Microwave Studio, until a suitable $50 \Omega$ broadband match was obtained, as shown in the smith chart in Fig. 3.

Fig. 3 HERE

The incident pulse used in the time domain simulation and the simulated probed pulse measured at $Z=+2 \mathrm{~mm}$ from the antenna, shown in Fig. 4, indicated that the device would exhibit the desired low-reverberation characteristic.

\section{Fig. 4 HERE}

Simulated peak power flow in the device clearly shows that unwanted reflections are largely subdued, as predicted by Wu King et al [7]; this is because injected energy is gradually absorbed along the length of the antenna, which in turn results in little or no reflected energy at the source port, Fig. 5.

\section{Fig.5 HERE}

It is also obvious from the simulated far field pattern, Fig.6, that the resistive profiling used results in very poor radiation efficiency $(\approx-30 \mathrm{~dB})$ and it is clear from this that such a device would only find use in short range applications or perhaps in those in which input power is not a limiting factor.

\section{Fig.6 HERE}

\section{ANTENNA FABRICATION}

A design based on an MBE grown GaAs layer on a high-resistivity $\mathrm{Si}$ substrate [14] was agreed upon in collaboration with FORTH [13], as this offered the quickest route to fabrication available to us at the time that allowed the growth and precise patterning of high- 
quality crystalline material ( $\mathrm{n}-\mathrm{GaAs}$ ), of a controlled resistivity, on a $\mathrm{Si}$ substrate. The monopole antenna, a diagrammatic representation of which is given in Fig.1, was designed using the parameters presented in Table 1 .

\section{Fig.1 HERE}

The computed values of all the design parameters used to fabricate the device are given in Table 2 and Fig. 2 shows the number of strips used to implement each segment's resistance.

\section{Table 2 HERE}

Two masks were purchased and used for Front-side and Back-side processing.

\section{Fig.7 HERE}

The monopoles were fabricated on the front-side of the MBE GaAs grown wafer $(50 \mathrm{~mm}$ in diameter) using conventional photolithography with two masks. The layout used, designed in Wavemaker [15], is shown in Figure 7.

The GaAs active areas were defined by etching away material with RIE (Reactive Ion Etching) down to the undoped GaAs buffer layer. The monopole's ohmic contacts were then deposited by electron gun evaporation of Au-Ge-Ni alloy and subsequent RTA (Rapid Thermal Anneal) in an inert atmosphere. The ground contact pads were also formed during this step.

The wafer was then processed on the back-side by thinning down to $370 \mu \mathrm{m}$ to match the simulated structure. It was then polished and a blanket deposition of aluminium was used to form the ground plane.

The devices were then completed by electrically connecting the back side metallization with the ground contact pads on the top of the wafer. Fig.8 and Fig.9 are SEM micrographs showing a close-up of an intersegment joint and the source metal to first segment overlap, respectively.

Fig.8 HERE

Fig.9 HERE

\section{Measured Results}

An Agilent PNA E8361A and Cascade 9000 manual probe-station were used to obtain a probed single-port sparameter measurement over $65 \mathrm{GHz}$, Fig. 10, of one of three available fabricated on-wafer devices. The graph indicates that the device response is indeed extremely wideband. The return loss shown is better than $8 \mathrm{~dB}$ ( $84 \%$ match) from $2.2 \mathrm{GHz}$ to above $65 \mathrm{GHz}$, the current upper limit of the system used. Also noteworthy is that the return loss improves to $\langle-15 \mathrm{~dB}$, for $45 \mathrm{GHz}\rangle$ $\mathrm{f}>60 \mathrm{GHz}$, with a decisive $>35 \mathrm{~dB}$ match at around $50 \mathrm{GHz}$.

\section{Fig.10 HERE}

A Picosecond pulse generator [16], Cascade manual probe station and Agilent 54750A 50GHz digitizing scope were used to measure the antenna's transient response to a $3.5 \mathrm{~V} 500 \mathrm{pS}$ pulse. A short electrical length $(4 \mathrm{~mm})$ near field probe was positioned $5 \mathrm{~mm}$ above the antenna using one of the probe-station's manipulators in order to receive the transmitted pulse. A reduced voltage injected pulse and actual probed transmitted pulse are shown in Fig.11 and Fig.12 respectively.

\section{Fig.11 HERE \\ Fig.12 HERE}

Fig.12 clearly shows that the antenna's response is practically non-reverberant, even without de-embedding the effects of the cabling used. The highest reverberation peak being at worst $(0.6 \mathrm{e}-3 / 2.8 \mathrm{e}-3) \times 100=21.4 \%$ of the transmitted pulse peak value, which is in broad accordance with other UWB antennas sporting the WuKing resistance profile [17, 18] [9] and is hence suitable for use in extremely broad-band time critical applications. However, the measured pulse has an amplitude of $2.5 \mathrm{mV}$ peak which is $.0025 / 3.5=1400 \mathrm{x}$ lower in amplitude than the injected pulse, indicating a loss of $63 \mathrm{~dB}$. These findings therefore indicate that the antenna's practical use will be limited to applications employing UWB scattering over a relatively short range, such as bio-target detection and monitoring / medical UWB radar. 


\section{ACKNOWLEDGMENT}

We are grateful to Dr George Konstantinidis and his team at FORTH (Greece) for their rapid monolithic prototyping service.

J. Hand is grateful for support from the NIHR Biomedical Research Centre funding scheme and we would also like to thank the UK Dept. Of Health's New and Emerging Applications of Technologies programme for funding project D007.

And we are also grateful for the filing of this work by Imperial Innovations Ltd. as UK Patent GB0800011.9.

\section{REFERENCES}

[1] Vilches, A.;Hand., J. Dispersion of UWB microwave pulses in tissues: a numerical study. in Annual Scientific Meeting of the Institute of Physics and Engineering in Medicine. 2005. University of Glasgow.

[2] Filonenko, E.L., Y; Hand, J;Vilches, A; Toumazou, C. Propagation of ultrawideband pulses within biological tissues. In Biodielectrics Theories, Mechanisms and Applications, 2006. Leicester, UK.

[3] Staderini, E.M., UWB Radars in Medicine. IEEE AESS Systems Magazine, 2002: p. 13-18.

[4] Sheppard, L.M., Not your mother's mammography [breast cancer detection]. Spectrum, IEEE, 2002. 39(10): p. 56-57.

[5] S.C. Hagness, A. Taflove, J.E. Bridges, Three-Dimensional FDTD analysis of pulsed microwave confocal system for breast cancer detection: design of an antenna array element. IEEE Trans. Antennas and Propagat., 1999. 47(5): p. 783-91.

[6] E.C. Fear, Xu Li, S.C. Hagness, M.A. Stuchly, Confocal microwave imaging for breast cancer detection: localization of tumors in three dimensions. Biomedical Engineering, IEEE Transactions on, 2002. 49(8): p. 812 - 822.

[7] KING, R.W.P; Wu, T.T., The Cylindrical Antenna with Nonreflecting Resistive Loading. IEEE Transactions on Antennas and Propagation, 1965. 13: p. 369-373.

[8] Maloney, J.G; Smith, Glenn S., A Study of Transient Radiation from the Wu-King Resistive Monopole-FDTD Analysis and Experimental Measurements. IEEE Transactions on Antennas and Propagation, 1993. 41(5): p. 668-676.

[9] Amert, T.W., J.; Albers, L.; Palecek, D.; Thompson, S.; Askildsen, B.; Whites, K.W.; Economical resistive tapering of bowtie antennas. Antennas and Propagation Society International Symposium, IEEE, 2004. 2: p. 1772 - 1775.

[10] CST, www.cst.com.

[11] Walter, M.M., I.;. FIT for EMC. in 17th International Zurich Symposium on Electromagnetic Compatibility. 2006. EMC-Zurich.

[12] Matlab, www.mathworks.com.

[13] FORTH, Found. for Research and Tech., Hellas (FORTH). Crete.: Dr. George Konstantinidis.

[14] Georgakilas, A.P., Ch.; Constantinidis, G; Tsagaraki, K; Krasny, H; Lochtermann, E. and Panayotatos, P., Effects of (100) Si Tilting Angle and Prelayer Conditions on GaAs/Si Heterostructures. Appl. Surf. Sci., 1996. 102: p. 67-72.

[15] Wavemaker, www.barnyardmicrosystems.com.

[16] Picosecond, www.picosecond.com.

[17] KANDA, M., A Relatively Short Cylindrical Broadband Antenna with Tapered Resistive Loading for Picosecond Pulse Measurements. IEEE Transactions on Antennas and Propagation, 1978. AP26(3).

[18] S.S.Stuchly, K.P.E., Pulse-receiving characteristics of resistively loaded dipole antennas. IEEE Transactions on Antennas and Propagation, 1990. 38(10): p. $1677-1688$. 


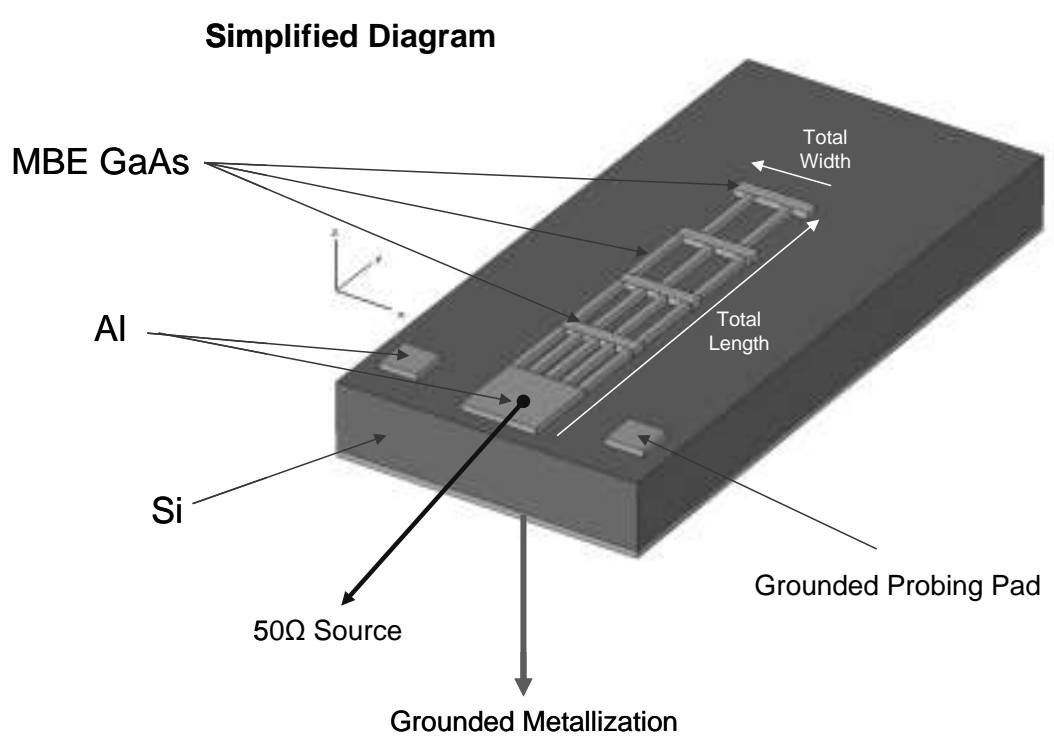

Figure 1, a very simplified diagram of the monolithic, segmented monopole. The feed point is the central pad shown with an arrow pointing to a $50 \mathrm{Ohm}$ source. 


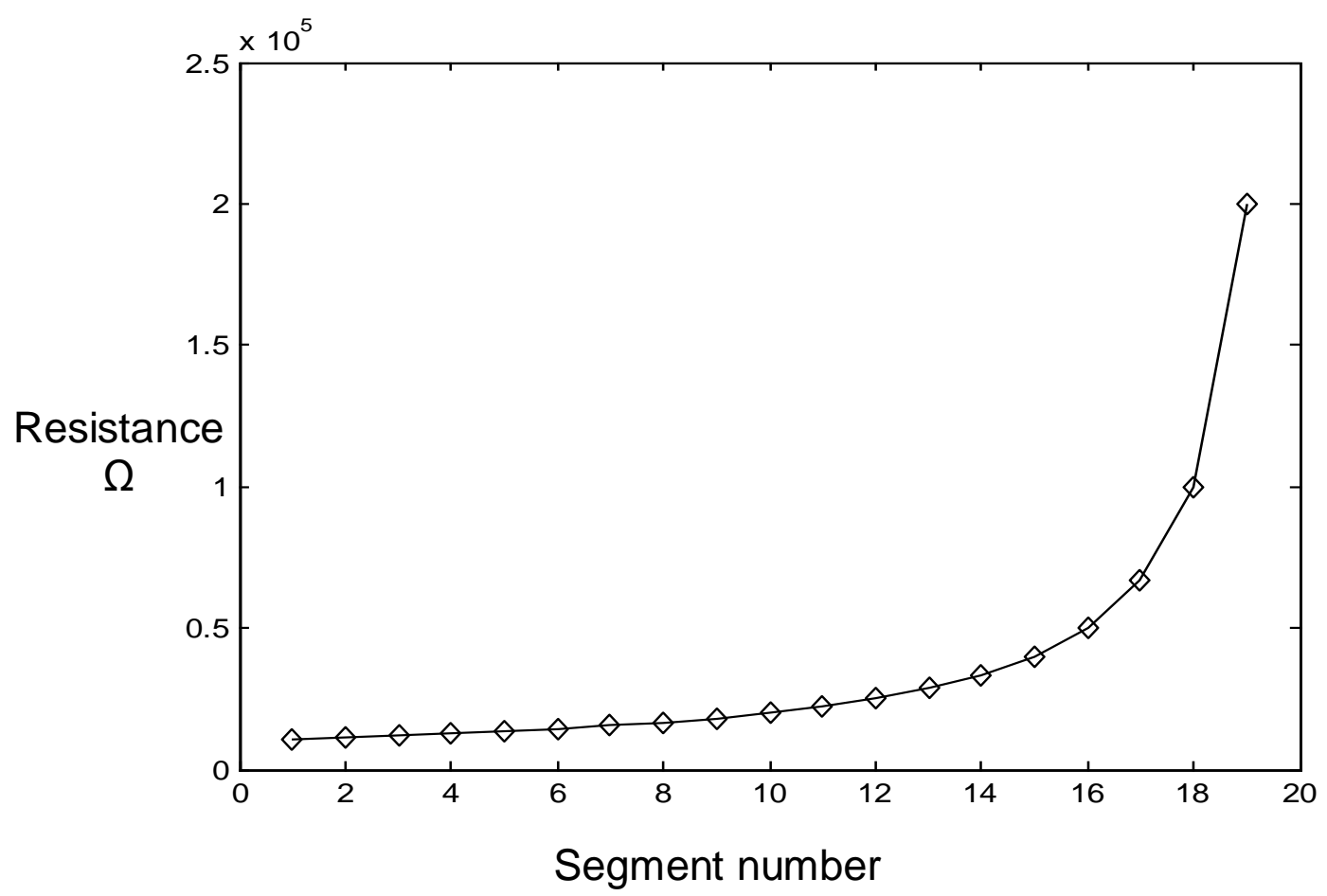

Figure 2, a typical segmented antenna resistive profile simulated in CST microwave studio. 


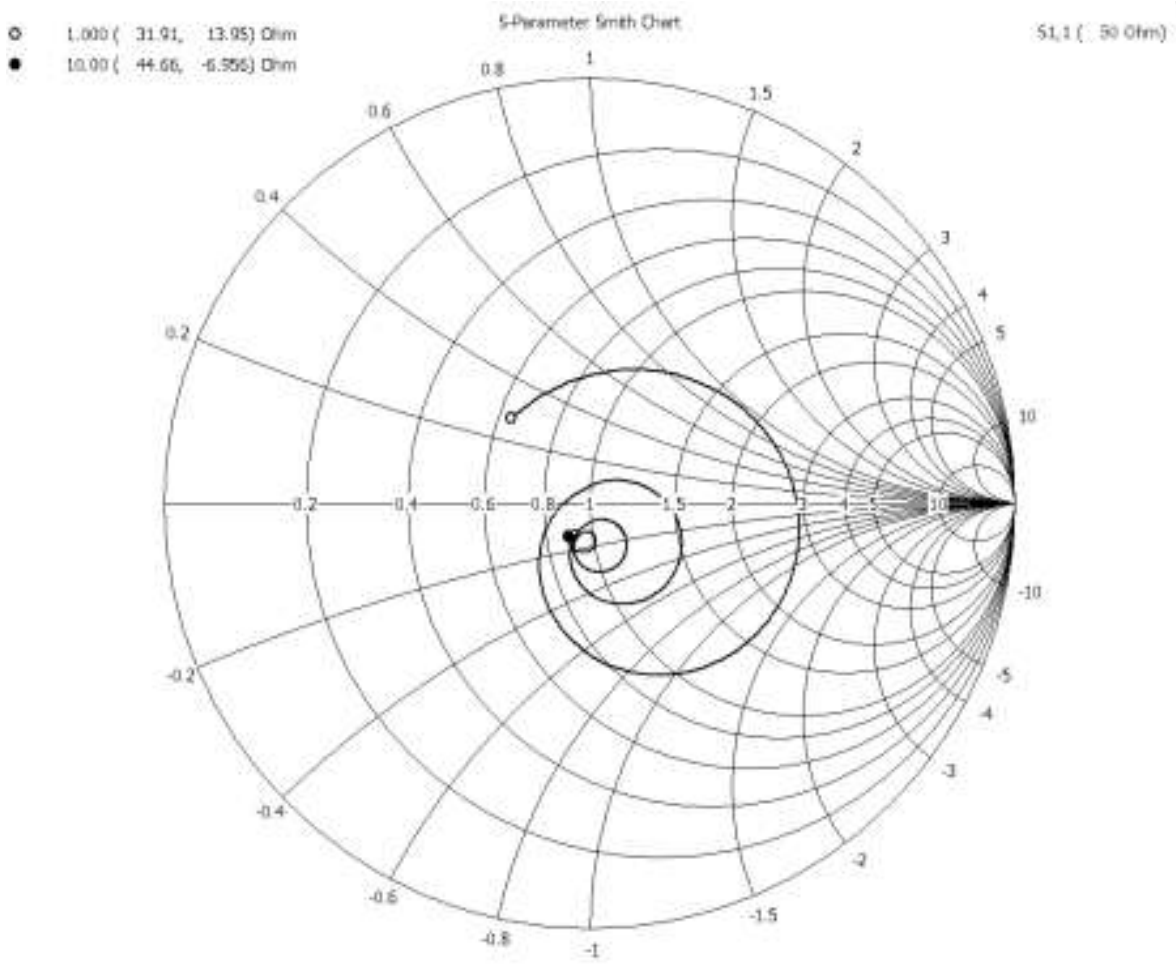

Figure 3, simulated Smith chart showing the return loss of the segmented antenna. 


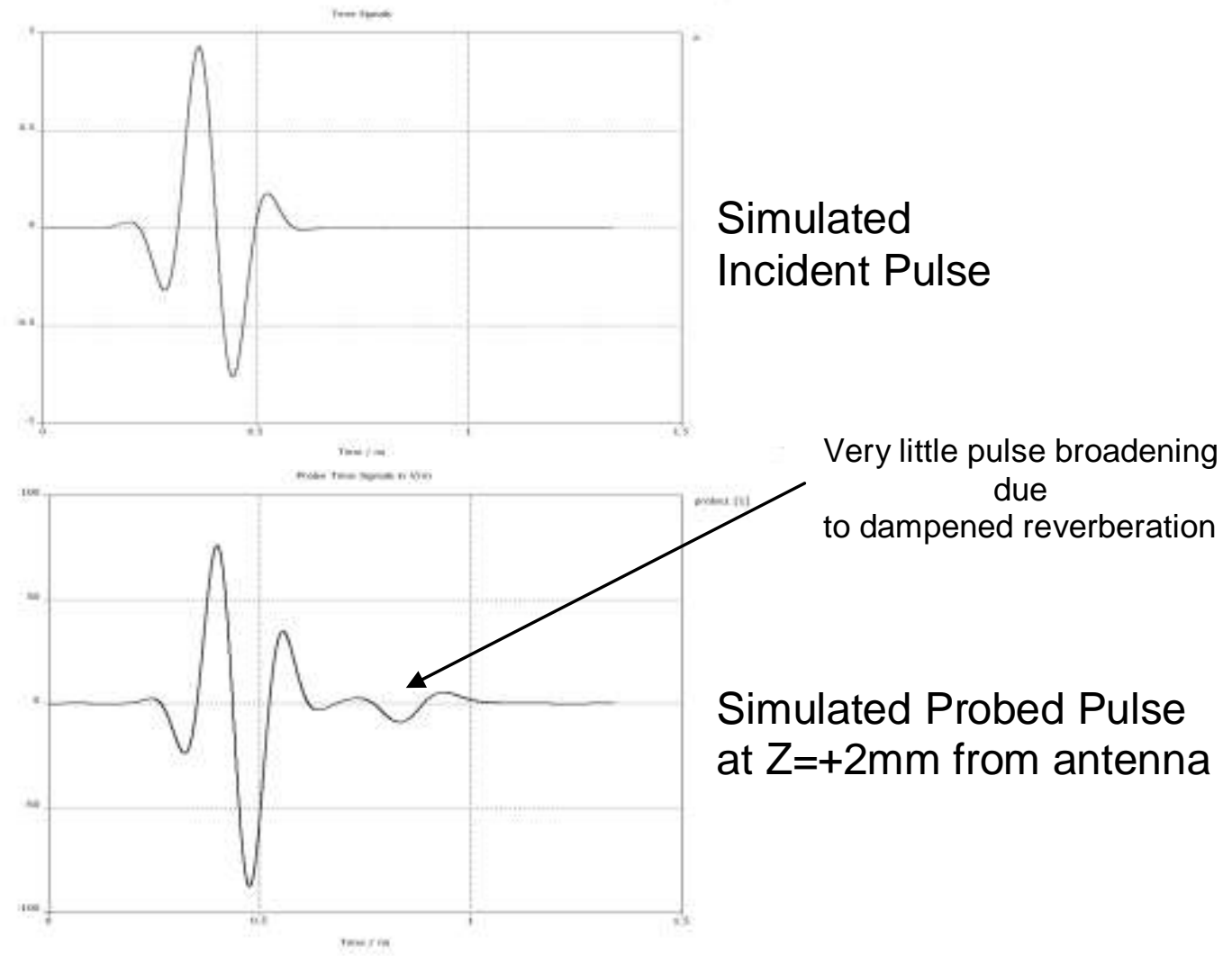

Figure 4, simulated incident vs probed 500pS pulses on a segmented antenna. 


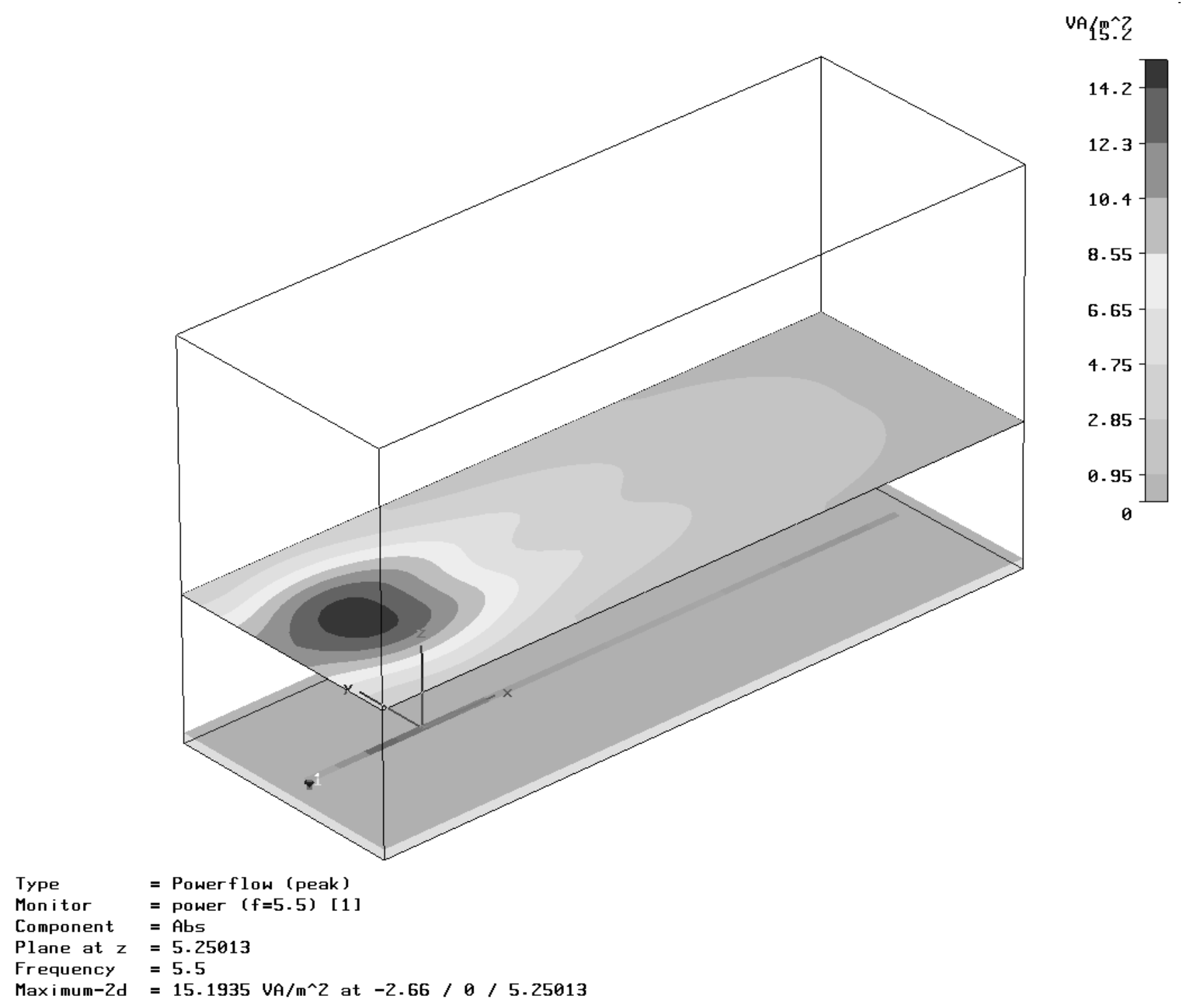

Figure 5, peak power flow at $\mathrm{Z}=+5.2 \mathrm{~mm}$ in the simulated antenna at $5.5 \mathrm{GHz}$. 


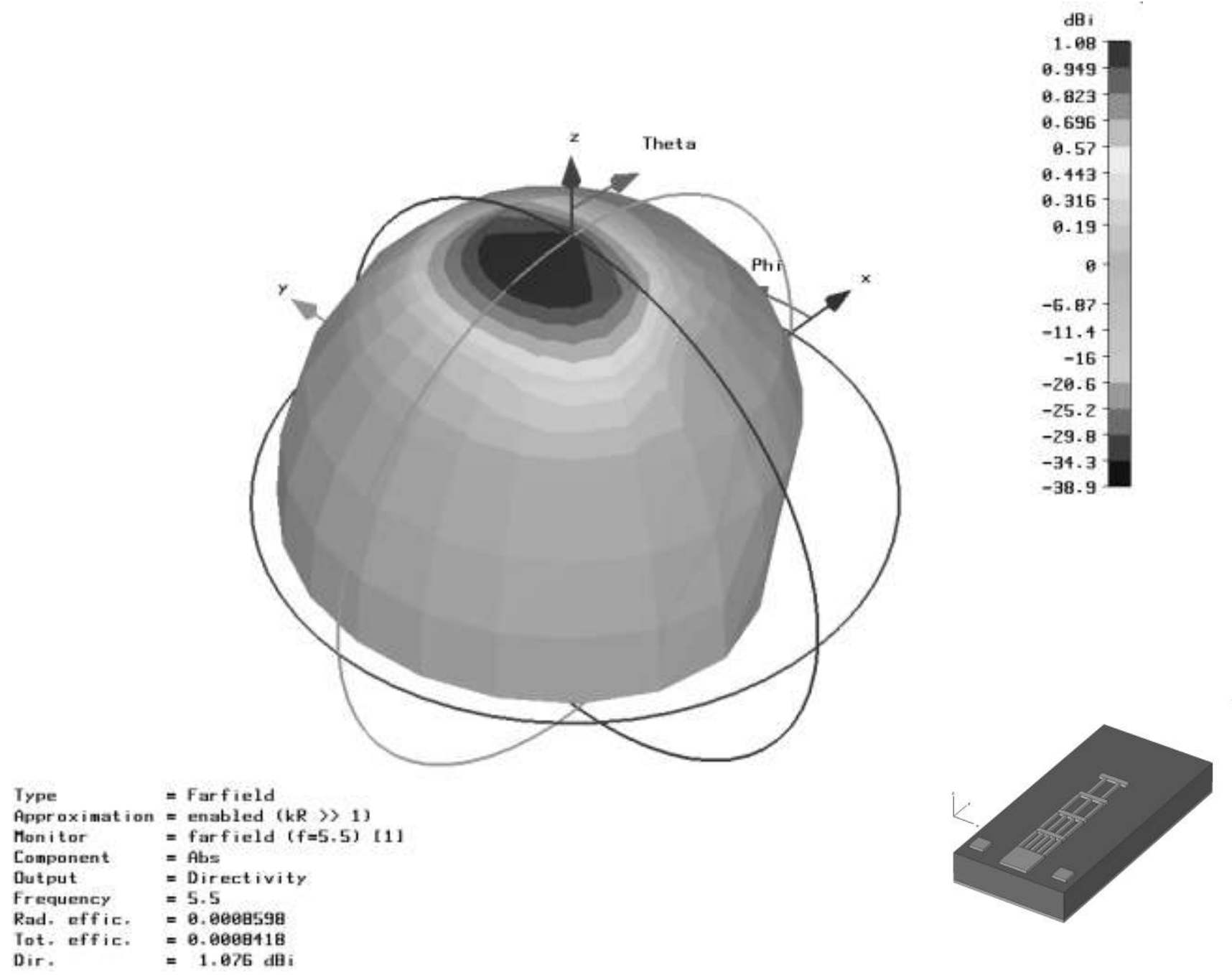

Figure 6, simulated far field pattern showing very poor radiation efficiency and $1 \mathrm{dBi}$ directivity in the $\mathrm{Z}$ direction. 


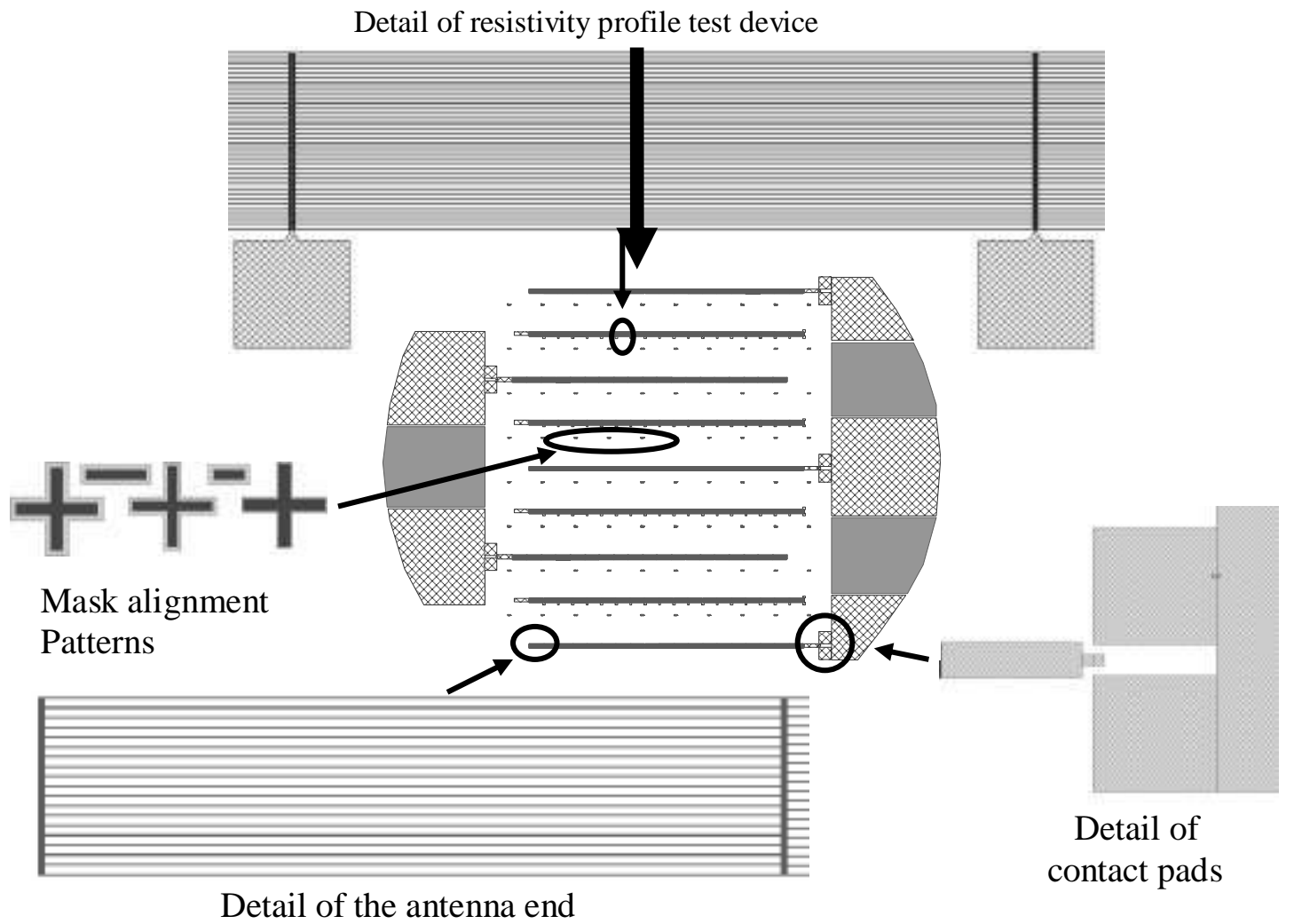

Figure 7, design mask layout of the prototype device wafer. Hatch pattern denotes metal. 


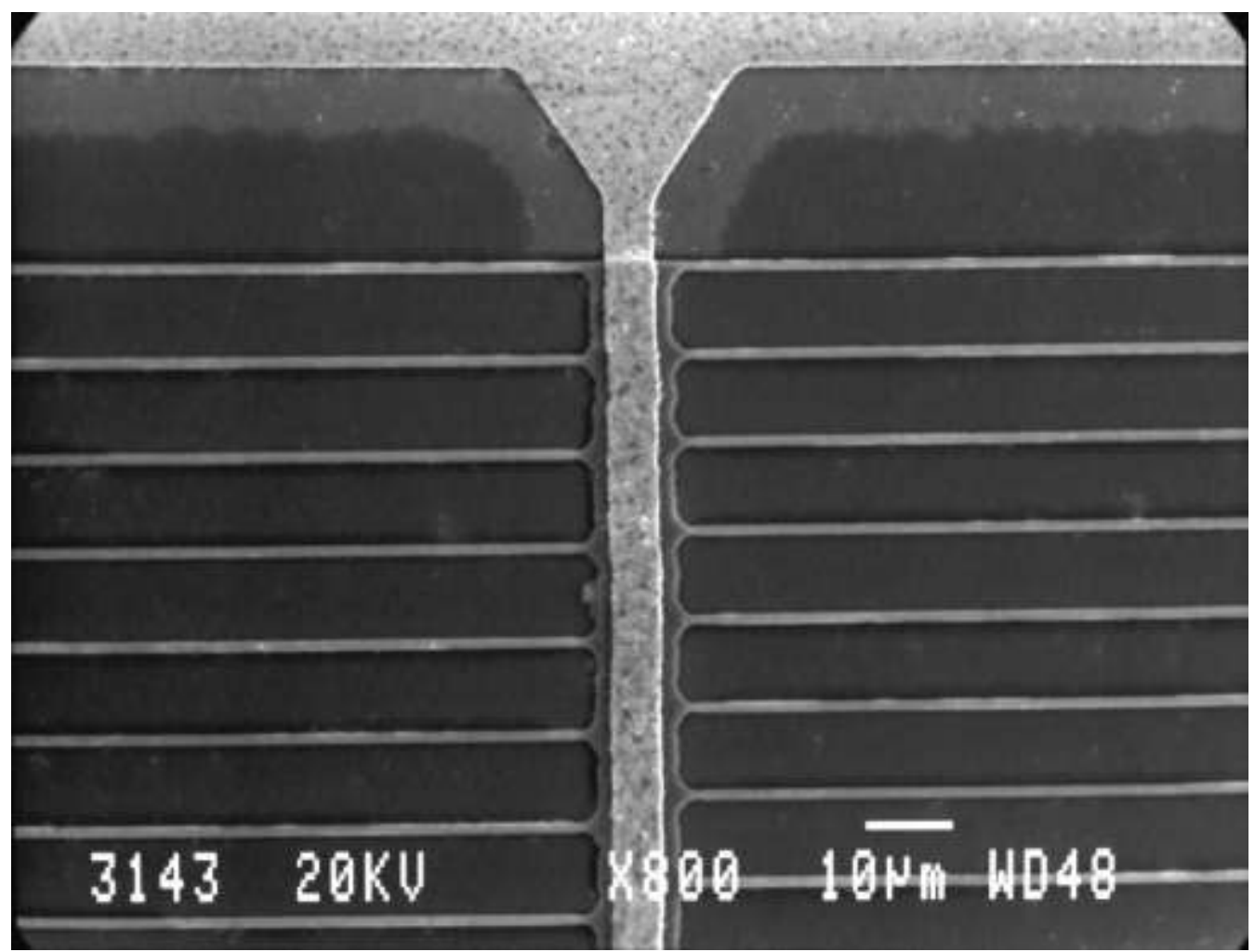

Figure 8, fabricated test device close-up of an inter-segment joint with ohmic contact for monitoring the resistivity profile. 


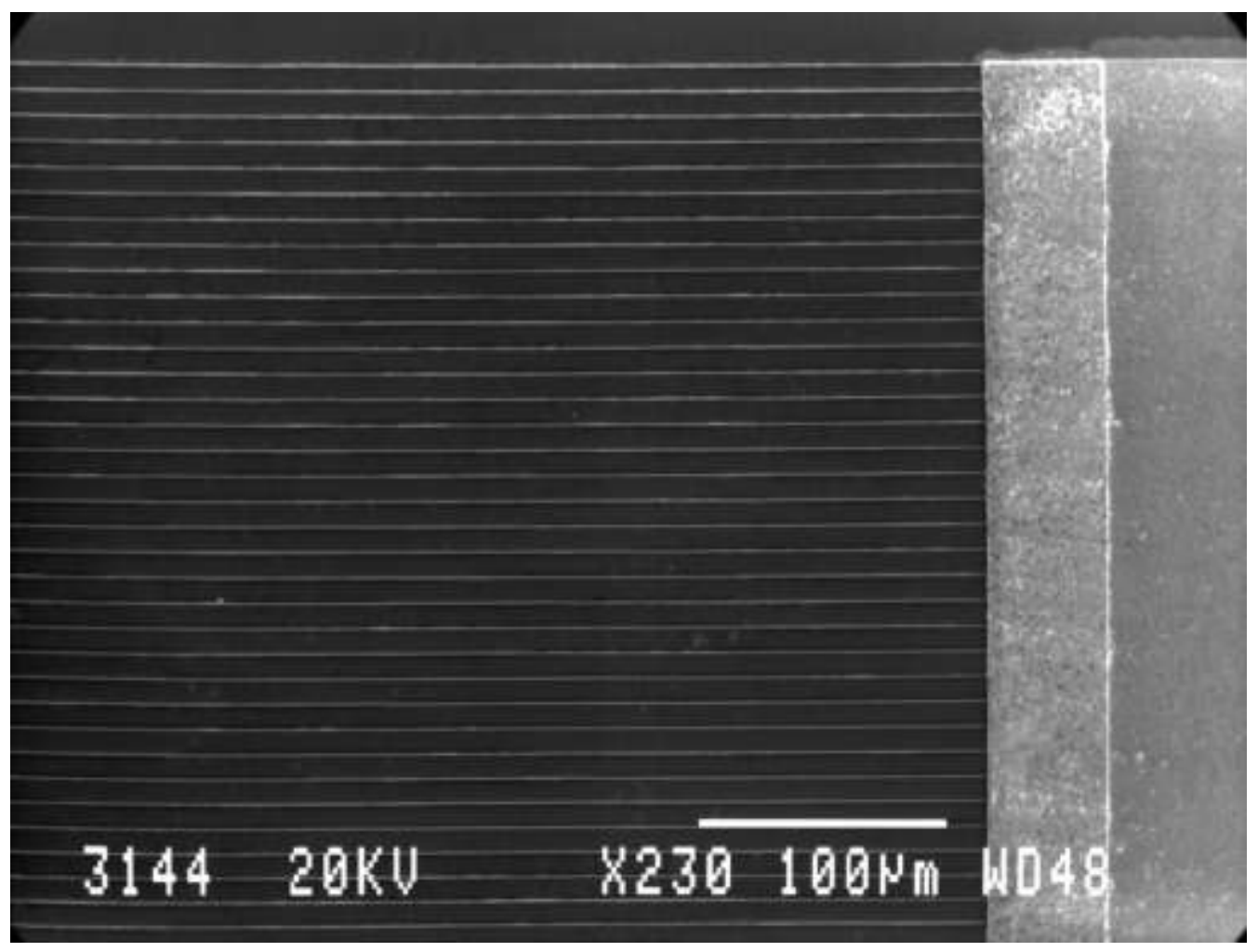

Figure 9, fabricated device source metal to first segment overlap. 


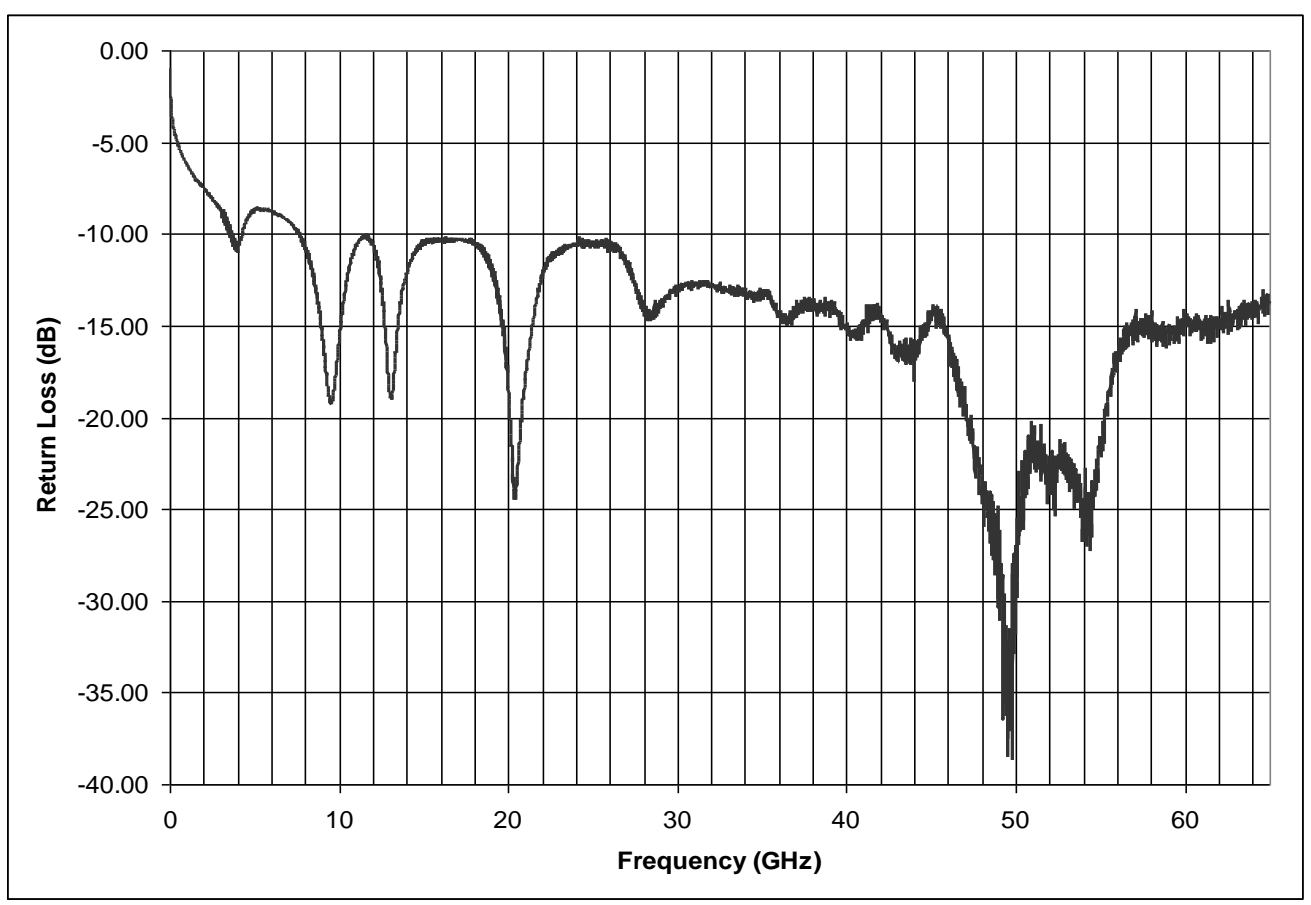

Figure 10, return loss $(10 \mathrm{MHz}$ to $65 \mathrm{GHz})$ from on-wafer probed device. 


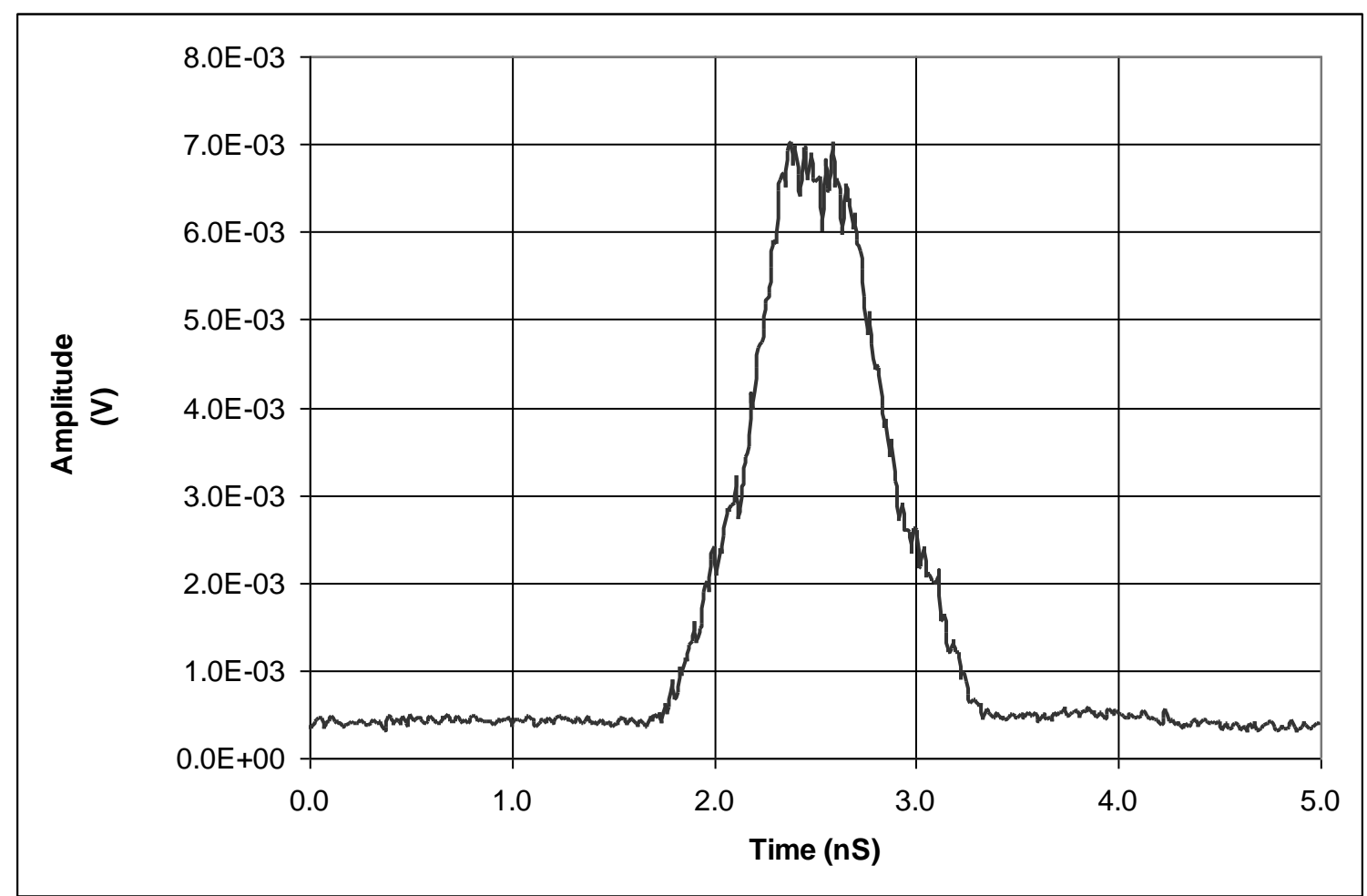

Figure 11, injected 500pS pulse measured by feeding pulse generator output straight into the oscilloscope via a short length of $50 \Omega$ SMA connected cable. Voltage was reduced for this measurement in order to avoid damaging the scope. 


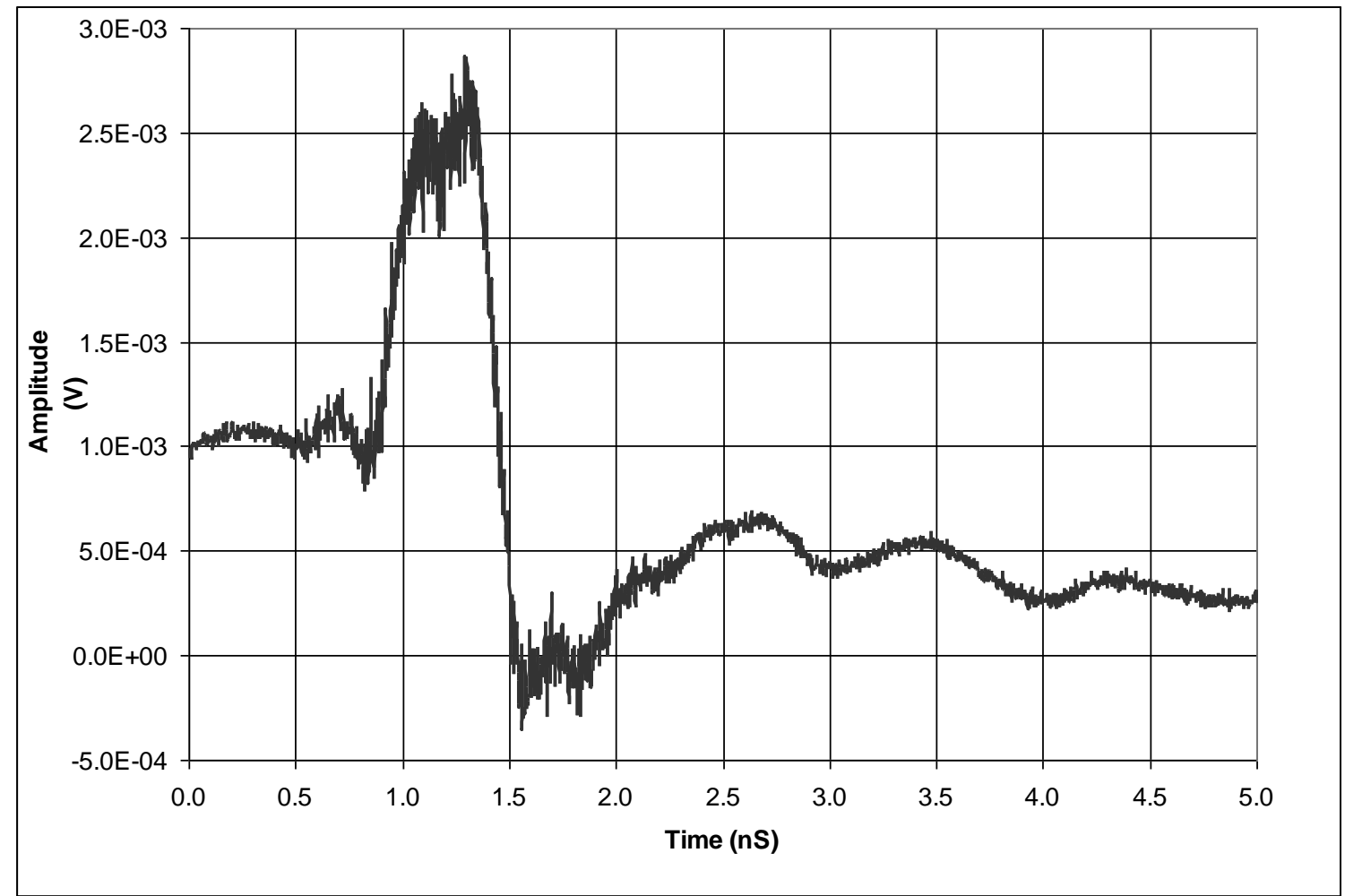

Figure 12, near field probed pulse, measured at $5 \mathrm{~mm}$ above the on-wafer device. 


\begin{tabular}{|}
$\begin{array}{c}\text { Lossy Si Substrate: } 28 \mathrm{~mm}(\mathrm{X}), 10 \mathrm{~mm}(\mathrm{Y}), 370 \mu \mathrm{m}(\mathrm{Z}), \\
\varepsilon \mathrm{r}=11.9, \operatorname{Tan} \delta=0.004\end{array}$ \\
\hline $\begin{array}{c}\text { Lossy MBE GaAs Buffer Layer: } 28 \mathrm{~mm}(\mathrm{X}), 10 \mathrm{~mm}(\mathrm{Y}), 1 \mu \mathrm{m} \\
(\mathrm{Z}), \varepsilon \mathrm{r}=12.9, \operatorname{Tan} \delta=0.006\end{array}$ \\
\hline MBE n-GaAs Segments: $1.3 \mathrm{~mm}(\mathrm{X}), 330 \mu \mathrm{m}(\mathrm{Y}), 0.5 \mu \mathrm{m}(\mathrm{Z})$, \\
$\rho=1.5 \mathrm{e}-4 \Omega . \mathrm{m}(150 \Omega / \square)$
\end{tabular}

Table 1, related technology parameters.

\begin{tabular}{|l||l|}
\hline Col.Width & $10 \mu \mathrm{m}$ \\
\hline Strip Height & $0.5 \mu \mathrm{m}$ \\
\hline Strip Width & $2 \mu \mathrm{m}$ \\
\hline Resistivity & $150 \mu \Omega . \mathrm{m}$ \\
\hline Col. Resistance & $18.182 \Omega$ \\
\hline Strip Resistance & $192 \mathrm{~K} \Omega$ \\
\hline
\end{tabular}

Table 2, related design parameters. 